\title{
ON THE MOTION OF NONVISCOUS COMPRESSIBLE FLUIDS IN DOMAINS WITH BOUNDARY
}

\author{
PAOLO SECCHI \\ Dipartimento di Matematica Pura ed Applicata \\ Università di Padova, via Belzoni 7, 35131 Padova, Italy
}

We want to present here some papers on the Euler equations of motion of nonviscous compressible fluid. We are interested in the solvability of these equations in the case of domains with boundary. The Euler equations are a typical initial-boundary value problem for a quasi-linear symmetric hyperbolic system; in the case of the usual solid wall boundary condition the boundary is characteristic. For this reason the solvability of these equations can be of more general interest, since the methods used for this problem can be applied also to other characteristic symmetric hyperbolic systems from mathematical physics, e.g. magnetohydrodynamics, shallow water,...

In fact, a theory for (even linear) symmetric hyperbolic mixed problems has been developed only for noncharacteristic boundaries (see [10]) or if the boundary matrices have constant rank near the boundary (see [9]).

For the sake of simplicity we shall consider only barotropic fluids, i.e. fluids for which the pressure is a function of the density only. The general case can be treated following the same ideas. The inviscid barotropic fluid motion in a domain $\Omega \subseteq \mathbb{R}^{3}$ is governed by the following equations (see Landau-Lifschitz [8]):

$$
\begin{aligned}
& \varrho[\dot{v}+(v \cdot \nabla) v-b]+\nabla p=0 \quad \text { in } Q_{T} \equiv(0, T) \times \Omega, \\
& \dot{\varrho}+\operatorname{div}(\varrho v)=0 \quad \text { in } Q_{T},
\end{aligned}
$$

where $\dot{v}=\partial v / \partial t,(v \cdot \nabla) v=\sum_{i} v_{i} \partial v / \partial x_{i}, \varrho=\varrho(t, x)$ is the unknown density, $v=\left(v_{1}, v_{2}, v_{3}\right)=v(t, x)$ is the unknown velocity field, $p=p(\varrho(t, x))$ is the unknown pressure of the fluid. For physical reasons $\varrho$ and $p^{\prime}(\varrho)$ are assumed to be positive. $b=\left(b_{1}, b_{2}, b_{3}\right)=b(t, x)$ is the given external force field.

For the initial value problem (i.e. $\Omega=\mathbb{R}^{3}$ ) we refer the reader to Kato [6], [7].

In the case of a bounded domain with a nonempty smooth boundary $\partial \Omega$ we 
are interested in, we prescribe the boundary condition

$$
v \cdot n=0 \quad \text { in } \Sigma_{T} \equiv(0, T) \times \partial \Omega
$$

where $n=n(x)$ is the outward normal vector to $\partial \Omega$.

Finally, the initial data at $t=0$ are given:

$$
\begin{aligned}
& v(0, x)=v_{0}(x), \\
& \varrho(0, x)=\varrho_{0}(x),
\end{aligned}
$$

where $\varrho_{0}(x)>0$ in $\bar{\Omega}$. Since the pressure is an increasing function of the density, we can invert this function and express the density as a known function of the pressure.

Considering velocity and pressure as unknowns (instead of velocity and density) has the advantage that (1) can be written as a quasi-linear symmetric system in $U=(v, p)$ :

$$
A_{0}(U) \frac{\partial U}{\partial t}+\sum_{j=1}^{3} A_{j}(U) \frac{\partial U}{\partial x_{j}}=F(U)
$$

where $A_{0}(U)$ is the diagonal positive definite matrix (here $\varrho^{\prime}=\varrho^{\prime}(p)$ )

$$
A_{0}(U)=\left(\begin{array}{cccc}
\varrho & & & \\
& \varrho & & \\
& & \varrho & \\
& & & \varrho^{\prime} / \varrho
\end{array}\right)
$$

$A_{j}(U)$ are the symmetric matrices

$$
A_{j}(U)=\left(\begin{array}{cccc}
\varrho v_{j} & & & \delta_{1 j} \\
& \varrho v_{j} & & \delta_{2 j} \\
& & \varrho v_{j} & \delta_{3 j} \\
\delta_{1 j} & \delta_{2 j} & \delta_{3 j} & \left(\varrho^{\prime} / \varrho\right) v_{j}
\end{array}\right)
$$

and $F(U)=(\varrho b, 0)$. The boundary matrix $A_{n}(U)$ is defined on $\partial \Omega$ as

$$
A_{n}(U):=\sum_{j=1}^{3} A_{j}(U) n_{j}=\left(\begin{array}{cc}
\varrho v \cdot n I_{3} & n \\
n^{T} & \left(\varrho^{\prime} / \varrho\right) v \cdot n
\end{array}\right)
$$

where $I_{3}$ is the $3 \times 3$ unit matrix. Because of (2), the boundary matrix is singular, namely the boundary is characteristic for (1), (2).

Hence the theory for noncharacteristic hyperbolic mixed problems (see [10]) cannot be applied. Even the theory for hyperbolic systems with rank of $A_{n}(U)$ uniformly constant near the boundary (see [9]) cannot be applied because the rank depends on the unknown $U$ and so it can vary arbitrarily close to the boundary.

In order to explain a little more this crucial point, let us try to solve (4), (2), (3) by means of the construction of successive approximations $U_{m}=\left(v_{m}, p_{m}\right)$ 
defined as the solutions of the linear problems

$$
\begin{cases}\frac{\partial U_{m}}{\partial t}+\sum_{j} A_{j}\left(U_{m-1}\right) \frac{\partial U_{m}}{\partial x_{j}}=F\left(U_{m-1}\right) & \text { in } Q_{T} \\ v_{m} \cdot n=0 & \text { on } \Sigma_{T} \\ U_{m}(0)=U_{m}^{(0)} & \text { in } \Omega .\end{cases}
$$

For the sake of simplicity here we assume that the positive definite matrix $A_{0}$ is the identity and that $\Omega=\mathbb{R}_{+}^{3}=\left\{x \in \mathbb{R}^{3}: x_{3}>0\right\}$. We have $n=(0,0,1)$, $A_{n}\left(U_{m-1}\right)=A_{3}\left(U_{m-1}\right)$.

The main point is to find a priori estimates such that $U_{m}$ belongs to a suitable invariant set. In order to obtain an a priori estimate in $L^{2}(\Omega)$ we multiply (8) by $U_{m}$ and integrate over $\Omega$. Integrating by parts gives

(9) $\frac{1}{2} \frac{d}{d t} \int\left|U_{m}\right|^{2}+\frac{1}{2} \int_{\partial \Omega} U_{m} A_{3} U_{m}-\frac{1}{2} \int U_{m} \sum_{j} \frac{\partial A_{j}}{\partial x_{j}} U_{m}=\int F\left(U_{m-1}\right) U_{m}$.

Since

$$
U_{m} A_{n} U_{m \mid \partial \Omega}=2 p_{m} v_{m} \cdot n_{\mid \partial \Omega}=0,
$$

if $\sum_{j} \partial A_{j} / \partial x_{j}$ is bounded on $Q_{T},(9)$ gives an $L^{2}$-estimate of the form

$$
\begin{aligned}
\left\|U_{m}(t)\right\|_{L^{2}(\Omega)} \leq & {\left[\left\|U_{m}(0)\right\|_{L^{2}(\Omega)}+\int_{0}^{t}\left\|F\left(U_{m-1}(\tau)\right)\right\|_{L^{2}(\Omega)} d \tau\right] } \\
& \times \exp \left(\frac{1}{2}\left\|\sum_{j} \frac{\partial A_{j}}{\partial x_{j}}\right\|_{L^{\infty}\left(Q_{T}\right)} T\right)
\end{aligned}
$$

for any $t \in[0, T]$. We now look for a priori estimates for derivatives of $U_{m}$. To do this, we differentiate $(8)_{1}$ and obtain a system whose principal part is the linear operator $\partial / \partial t+\sum_{j} A_{j}\left(U_{m-1}\right) \partial / \partial x_{j}$ applied to the derivatives of $U_{m}$. Taking a partition of unity in the domain we consider separately interior estimates and estimates near the boundary. Interior a priori estimates can be obtained in a straightforward way.

To avoid higher order derivatives it is necessary to integrate by parts; this can be done without problems since no boundary integral arises.

To obtain a priori estimates near the boundary we observe that computations similar to the ones for the $L^{2}$-estimate can be done for tangential and time derivatives.

In fact, such derivatives obey the same boundary condition $(8)_{2}$, as we see by differentiating $(8)_{2}$ with respect to time or tangential directions.

Difficulties arise with normal derivatives for which no boundary condition can be obtained by differentiating $(8)_{2}$. Normal derivatives can be obtained from $(8)_{1}$ 
written in the form

$$
A_{3} \frac{\partial U_{m}}{\partial x_{3}}=-\left[\frac{\partial U_{m}}{\partial t}+\sum_{j=1}^{2} A_{j} \frac{\partial U_{m}}{\partial x_{j}}\right]+F .
$$

If the boundary matrix is nonsingular, it can be inverted and from (11) one can express the normal derivatives $\partial U_{m} / \partial x_{3}$ by means of time and tangential derivatives already estimated.

Unfortunately, this is not our case since the boundary is characteristic.

Since no known theory covers our case, it is necessary to introduce some special approach to prove solvability of (1)-(3).

A first contribution was given by D. Ebin in [5], where he proved the existence for short time of a regular solution to (1)-(3) provided that the initial velocity is subsonic and the initial density is nearly constant (and $b \equiv 0$ ).

The existence of a solution to (1)-(3) for general initial data was then proved by H. Beirão da Veiga [2], [3] and R. Agemi [1]. Denote by $X_{r}^{s}\left(T, \Omega, \mathbb{R}^{m}\right)(r<s$ positive integers) the space of functions (simply denoted by)

$$
X_{r}^{s}:=\bigcap_{k=0}^{s-r} C^{k}\left([0, T], H^{s-k}\left(\Omega, \mathbb{R}^{m}\right)\right)
$$

where $H^{s}\left(\Omega, \mathbb{R}^{m}\right)$ is the set of $\mathbb{R}^{m}$-valued functions on $\Omega$ in the Sobolev space $H^{s}$ and $C^{k}\left([0, T], H^{s-k}\left(\Omega, \mathbb{R}^{m}\right)\right)$ is the space of bounded $k$ times continuously differentiable functions on $[0, T]$ with values in $H^{s-k}\left(\Omega, \mathbb{R}^{m}\right)$. We set $X_{0}^{s}=X^{s}$.

Their result is the following:

TheOREM 1 ([2], [3], [1]). Suppose that the pressure $p \in C^{s+1}(\mathbb{R}), s \geq 3$, and that the data $\left(v_{0}, \varrho_{0}, b\right)$ belong to $H^{s}\left(\Omega, \mathbb{R}^{4}\right) \times X_{1}^{s}\left(T_{0}, \Omega, \mathbb{R}^{3}\right), T_{0}>0, \varrho_{0}>0$ in $\bar{\Omega}$, and satisfy the necessary compatibility conditions up to order $s$. Then there exists a positive constant $T \leq T_{0}$ such that $(1)-(3)$ has a unique solution $(v, \varrho)$ in $X^{s}\left(T, \Omega, \mathbb{R}^{4}\right)$, such that $\varrho(t, x)>0$ in $[0, T] \times \bar{\Omega}$.

R emark. H. Beirão da Veiga considers only the case $s=3$. Following the same method it is possible to obtain the result for $s \geq 3$.

Plan of the proof. By taking account of the special form of the equations, system (1)-(3) is solved by transforming it into equivalent problems. In [2], [3] the following systems are considered (for the sake of simplicity assume $\Omega$ to be simply connected):

$$
\begin{gathered}
\begin{cases}\operatorname{div} v=\theta & \text { in } Q_{T}, \\
\operatorname{rot} v=\xi & \text { in } Q_{T}, \\
v \cdot n=0 & \text { on } \Sigma_{T},\end{cases} \\
\begin{cases}\dot{\xi}+(v \cdot \nabla) \xi-(\xi \cdot \nabla) v+\theta \xi=\operatorname{rot} b & \text { in } Q_{T}, \\
\xi(0)=\operatorname{rot} v_{0} & \text { on } \Sigma_{T},\end{cases}
\end{gathered}
$$


and, setting $g=\log \varrho, h(g)=p^{\prime}\left(e^{g}\right)$,

$$
\begin{cases}\dot{\theta}+v \cdot \nabla \theta+\operatorname{div}[h(g) \nabla g]=\operatorname{div} b-\sum_{i j} D_{i} v_{j} D_{j} v_{i} & \text { in } Q_{T}, \\ \dot{g}+v \cdot \nabla g+\theta=0 & \text { in } Q_{T}, \\ \theta(0)=\operatorname{div} v_{0} & \text { on } \Omega, \\ g(0)=\log \varrho_{0} & \text { on } \Omega, \\ h(g) \frac{\partial g}{\partial n}=\sum_{i j}\left(D_{i} n_{j}\right) v_{i} v_{j}+b \cdot n & \text { on } \Sigma_{T} .\end{cases}
$$

System (12)-(14) is equivalent to (1)-(3). Denoting by $V$ the left-hand side of $(1)_{1}$ divided by $\varrho$ and using (12) we see that $(13)_{1}$ is $\operatorname{rot} V=0$ in $Q_{T},(14)_{1}$ is $\operatorname{div} V=0$ in $Q_{T}$ and $(14)_{5}$ is $V \cdot n=0$ on $\Sigma_{T}$. The three equations hold if and only if $V=0$ in $Q_{T}$, that is, $(1)_{1}$ is satisfied. (1) $)_{2}$ is given by $(14)_{2}$.

System (12)-(14) is solved by means of a fixed point argument. First, consider system $(12)_{\delta, \zeta}$, that is, $(12)_{\theta, \xi}$ with $(\theta, \xi)$ replaced by some given $(\delta, \zeta)$. This system is elliptic and gives a velocity $v$. Secondly, consider $(13)_{\delta}$, that is, $(13)_{\theta}$ with $\theta$ replaced by $\delta$ and $v$ obtained from $(12)_{\delta, \zeta}$. (13) $\delta$ is a linear first order hyperbolic equation that can be solved by means of the method of characteristics. Given $\delta$, one can show the existence of a fixed point of the map $\zeta \rightarrow \xi$, provided that $T$ is sufficiently small.

Consider now system $(14)_{q}$, that is, $(14)_{g}$ with $h(g)$ replaced by $h(q)$ and $v$ the velocity corresponding to the fixed point $\zeta=\xi$. To solve $(14)_{q}$, apply the operator $\partial / \partial t+v \cdot \nabla$ to equation $(14)_{2}$. Combining with $(14)_{1}$ we have

$$
\left(\frac{\partial}{\partial t}+v \cdot \nabla\right)^{2} g-\operatorname{div}[h(q) \nabla g]=\sum_{i, j} D_{i} v_{j} D_{j} v_{i}-\operatorname{div} b \quad \text { in } Q_{T} .
$$

To equation (15) we add the boundary condition $(14)_{q, 5}$ and the initial conditions $(14)_{4}$ and

$$
\dot{g}(0)=-v_{0} \cdot \nabla g(0)-\operatorname{div} v_{0},
$$

obtained from $(14)_{2}$ calculated at time $t=0$. System $(15),(14)_{5},(14)_{4},(16)$ is a linear hyperbolic mixed problem of second order.

Given the solution $g$ we finally find $\theta=-\dot{g}-v \cdot \nabla g$. If $T$ is sufficiently small the map $(\delta, q) \rightarrow(\theta, g)$ has a fixed point, i.e. a solution of $(12)_{\theta, \xi},(13)_{\theta},(14)_{g}$.

Agemi's equivalent formulation is similar to Ebin's. The density is again obtained from $(15),(14)_{5},(14)_{4},(16)$, while for the velocity the Helmholtz decomposition of a vector field into its soleinodal part and its gradient part is introduced. The two components of this decomposition of $v$ are obtained as solutions of two suitable problems.

Another interesting contribution to the argument is Schochet's paper [11], where a nonisentropic compressible fluid in a bounded domain is considered (with $b \equiv 0$ ). A short-time existence theorem is proved for general initial data, apart 
from regularity assumptions and the necessary compatibility conditions. Furthermore, solutions with low Mach number and almost incompressible initial data are shown to be close to corresponding solutions of the equations for incompressible flow.

The solvability of the equations is not obtained by solving equivalent problems as before. The solution is constructed as the limit of the solutions of approximating equations, for which the boundary is no more characteristic, so that the theory for noncharacteristic quasi-linear symmetric hyperbolic problems can be applied.

The crucial point is how to prove uniform a priori estimates for the approximating solutions and, in particular, as already pointed out, how to estimate the normal derivatives. Apart from technical difficulties the main points are the following. Assume for the sake of simplicity that $\Omega=\mathbb{R}_{+}^{3}$. On $\partial \Omega=\left\{x_{3}=0\right\}$ the boundary matrix $A_{3}$ is

$$
\left(\begin{array}{lllll} 
& & & 0 \\
& O_{3} & & 0 \\
& & & 1 \\
0 & 0 & 1 & 0
\end{array}\right)
$$

of rank two. In a thin layer of $\partial \Omega$ the rank is still at least two, due to the continuity of $U_{m-1}$ (the approximating solution at step $m-1$ ). From (11) we can obtain, inverting the last two rows, the normal derivatives $\partial v_{3} / \partial x_{3}, \partial p / \partial x_{3}$ in terms of time and tangential derivatives and of the other normal derivatives $\partial v_{1} / \partial x_{3}$, $\partial v_{2} / \partial x_{3}$ (for simplicity we drop the index $m-1$ ). From a vorticity equation similar to (13), one can then obtain an estimate for $\xi=\operatorname{rot} v$ because of the fact that the vorticity equation (like (13)) is of transport type (this implies that also normal derivatives of $\xi$ can be estimated). Finally, it is shown that the usual norm in the space $X^{3}$ (where the solution is sought) is equivalent to the sum of a norm including derivatives up to order three but no normal derivative of third order, plus the norm in $X^{2}$ of the normal derivatives $\partial v_{3} / \partial x_{3}, \partial p / \partial x_{3}$, plus the norm in $X^{2}$ of rot $v$. Then the estimates for the two normal derivatives $\partial v_{3} / \partial x_{3}, \partial p / \partial x_{3}$, for the vorticity $\xi$ and the standard estimates for the tangential derivatives allow us to obtain the a priori estimate in $X^{3}$. For all the details see [11].

This procedure is improved in [12] as shown below.

In [12] I study the motion of a nonviscous compressible barotropic fluid in a time-dependent domain $\Omega_{t}$ of the three-dimensional space. For each $t \in\left[0, T_{0}\right]$, $T_{0}>0, \Omega_{t}$ is defined as $\Omega_{t}:=\mu(t, \Omega)$ where $\mu:\left[0, T_{0}\right] \times \bar{\Omega} \rightarrow \mathbb{R}^{3}$ is a given smooth map such that $\mu(0, \Omega)=\Omega$ and such that $\mu(t, \cdot): \Omega \rightarrow \Omega_{t}$ is a diffeomorphism, for each $t \in\left[0, T_{0}\right]$. The equations of motion (1) are to be satisfied in the noncylindrical domain $D_{T}:=\bigcup_{0<t<T}\{t\} \times \Omega_{t}$ :

$$
\begin{gathered}
\varrho[\dot{v}+(v \cdot \nabla) v-b]+\nabla p=0 \quad \text { in } D_{T}, \\
\dot{\varrho}+\operatorname{div}(\varrho v)=0 \quad \text { in } D_{T},
\end{gathered}
$$


the boundary condition (2) is replaced by

(19) $u(t, y) \cdot n^{t}(y)=\dot{\mu}(t, x) \cdot n^{t}(y), \quad t \in(0, T), y=\mu(t, x) \in \partial \Omega_{t}, x \in \partial \Omega$,

where $n^{t}$ is the outward normal vector to $\partial \Omega_{t}$. (19) states that there is no flux through the moving boundary. Finally, we add the initial conditions (3).

In the particular case $\mu(t, \cdot)=$ Id (identity on $\bar{\Omega}$ ), problem (18), (19), (3) reduces to $(1)-(3)$.

We reduce problem (18), (19), (3) to the cylindrical domain $Q_{T}=(0, T) \times \Omega$ by the change of variable $(t, y) \rightarrow(t, x)$, where $x$ is such that $y=\mu(t, x)$. Set

$$
\bar{p}(t, x):=p(t, y)=p(t, \mu(t, x)), \quad \bar{u}(t, x):=v(t, \mu(t, x))-\dot{\mu}(t, x)
$$

and denote by $A=A(t, x)$ the transpose $\left(a_{k i}\right)^{T}$ of the jacobian matrix $[D \mu]^{-1}=$ $\left(a_{k i}\right)$. Then we have the transformed problem (we drop the bar in $\bar{p}, \bar{u}$ )

$$
\begin{cases}\varrho(p)[\dot{u}+(u \cdot A \nabla) u+(u \cdot A \nabla) \dot{\mu}+\ddot{\mu}-b]+A \nabla p=0 & \text { in } Q_{T}, \\ \left(\varrho^{\prime} / \varrho\right)[\dot{p}+u \cdot A \nabla p]+A \nabla \cdot u+A \nabla \cdot \dot{\mu}=0 & \text { in } Q_{T}, \\ u \cdot N=0 & \text { on } \Sigma_{T}, \\ u(0)=v_{0}-\dot{\mu}(0) & \text { in } \Omega, \\ p(0)=p\left(\varrho_{0}\right) & \text { in } \Omega,\end{cases}
$$

where $N(t, x):=n^{t}(\mu(t, x))$.

For problem (20) we prove the existence for short time and uniqueness of a regular solution (a result similar to the one of Theorem 1) and by means of this result the existence of classical solutions to problem (18), (19), (3). As in [11] the solution is constructed as the limit of solutions to approximating equations, for which the boundary is not characteristic so that the theory for noncharacteristic quasi-linear symmetric hyperbolic systems can be applied. To explain how we get the estimates for the normal derivatives, we write (20) in vector form as

$$
A_{0}(U) \frac{\partial U}{\partial t}+\sum_{j=1}^{3} A_{j}(U) \frac{\partial U}{\partial x_{j}}+D(U) U=F(U),
$$

where $U=(u, p), A_{0}$ is given by (5),

$$
\begin{gathered}
A_{j}(U)=\left(\begin{array}{cccc}
\varrho v_{i} a_{j i} & 0 & 0 & a_{j 1} \\
0 & \varrho v_{i} a_{j i} & 0 & a_{j 2} \\
0 & 0 & \varrho v_{i} a_{j i} & a_{j 3} \\
a_{j 1} & a_{j 2} & a_{j 3} & \left(\varrho^{\prime} / \varrho\right) v_{i} a_{j i}
\end{array}\right), \\
D(U)=\varrho(p)\left(\begin{array}{cccc}
a_{j 1} D_{j} \dot{\mu}_{1} & a_{j 2} D_{j} \dot{\mu}_{1} & a_{j 3} D_{j} \dot{\mu}_{1} & 0 \\
a_{j 1} D_{j} \dot{\mu}_{2} & a_{j 2} D_{j} \dot{\mu}_{2} & a_{j 3} D_{j} \dot{\mu}_{2} & 0 \\
a_{j 1} D_{j} \dot{\mu}_{3} & a_{j 2} D_{j} \dot{\mu}_{3} & a_{j 3} D_{j} \dot{\mu}_{3} & 0 \\
0 & 0 & 0 & 0
\end{array}\right),
\end{gathered}
$$

where the summation convention over repeated indices is assumed,

$$
F(U)=(\varrho(p)(b-\ddot{\mu}),-A \nabla \cdot \dot{\mu}) .
$$


The boundary matrix is

$$
A_{n}(U)=\left(\begin{array}{cc}
\varrho u \cdot A n I_{3} & A n \\
(A n)^{T} & \left(\varrho^{\prime} / \varrho\right) u \cdot A n
\end{array}\right) .
$$

Assuming for the sake of simplicity that $\Omega=\mathbb{R}_{+}^{3}, A_{n}(U)$ reduces to (17) on $\partial \Omega$ at time $t=0$ (recall that $A(0)=I_{3}$ ). Hence, for short time, in a thin layer of $\partial \Omega$, the last two rows are linearly independent. On the other hand, as in the case of the fixed-in-time domain, one can consider a vorticity equation (similar to (13)) and obtain an a priori estimate in $X^{2}$ for the transformed vorticity $\widetilde{\xi}=A \nabla \wedge u$.

We have

$$
\widetilde{\xi}_{1}=a_{k 2} \frac{\partial u_{3}}{\partial x_{k}}-a_{k 3} \frac{\partial u_{2}}{\partial x_{k}}, \quad \widetilde{\xi}_{2}=a_{k 3} \frac{\partial u_{1}}{\partial x_{k}}-a_{k 1} \frac{\partial u_{3}}{\partial x_{k}},
$$

where the summation convention is assumed. We can isolate the normal derivatives $\partial u / \partial x_{3}$ in (21) to obtain

$$
a_{32} \frac{\partial u_{3}}{\partial x_{3}}-a_{33} \frac{\partial u_{2}}{\partial x_{3}}=\widetilde{\xi}_{1}+\text { "tan", } \quad a_{33} \frac{\partial u_{1}}{\partial x_{3}}-a_{31} \frac{\partial u_{3}}{\partial x_{3}}=\widetilde{\xi}_{2}+\text { "tan", }
$$

where "tan" contains the tangential derivatives of (21). Consider now the linear system with unknown $\partial U / \partial x_{3}$ formed by (22) and the third and fourth row of

$$
A_{3} \frac{\partial U}{\partial x_{3}}=-\left[A_{0} \frac{\partial U}{\partial t}+\sum_{j=1}^{2} A_{j} \frac{\partial U}{\partial x_{j}}+D U\right]+F .
$$

The linear system is

$$
\Lambda \frac{\partial U}{\partial x_{3}}=\left(\widetilde{\xi}_{1}, \widetilde{\xi}_{2}, 0,0\right)+\text { "tan" }
$$

where "tan" contains only time and tangential derivatives and normal derivatives of lower order or given terms; the matrix $\Lambda$ of coefficients is

$$
\Lambda=\left(\begin{array}{cccc}
0 & -a_{33} & a_{32} & 0 \\
a_{33} & 0 & -a_{31} & 0 \\
0 & 0 & \varrho u_{i} a_{3 i} & a_{33} \\
a_{31} & a_{32} & a_{33} & \left(\varrho^{\prime} / \varrho\right) u_{i} a_{3 i}
\end{array}\right)
$$

whose determinant on $\partial \Omega$ at time $t=0$ is equal to -1 . Hence in a thin layer of $\partial \Omega$, for short time, $\operatorname{det} \Lambda$ is different from zero and we can obtain from $(23)$ all the normal derivatives in terms of the transformed vorticity $\widetilde{\xi}$, of time and tangential derivatives and of lower order terms. This permits us to obtain the a priori estimates for the approximating solutions. For all the details see [12].

Other interesting papers related to the subject are quoted below.

\section{References}

[1] R. Agemi, The initial boundary value problem for inviscid barotropic fluid motion, Hokkaido Math. J. 10 (1981), 156-182. 
[2] H. Beirão da Veiga, Un théorème d'existence dans la dynamique des fluides compressibles, C. R. Acad. Sci. Paris 289 B (1979), 297-299.

[3] -, On the barotropic motion of compressible perfect fluids, Ann. Scuola Norm. Sup. Pisa 8 (1981), 317-351.

[4] -, Homogeneous and non-homogeneous boundary value problems for first order linear hyperbolic systems arising in fluid mechanics, Comm. Partial Differential Equations, part I: 7 (1982), 1135-1149, part II: 8 (1983), 407-432.

[5] D. Ebin, The initial boundary value problem for subsonic fluid motion, Comm. Pure Appl Math. 32 (1979), 1-19.

[6] T. Kato, Quasi-linear equations of evolution, with applications to partial differential equations, in: Spectral Theory and Differential Equations, Lecture Notes in Math. 448, Springer, 1975, 25-70.

[7] - , The Cauchy problem for quasi-linear symmetric hyperbolic systems, Arch. Rational Mech. Anal. 58 (1975), 181-205.

[8] L. Landau et E. Lifschitz, Mécanique des fluides, Mir, Moscou 1971.

[9] A. Majda and S. Osher, Initial-boundary value problems for hyperbolic equations with uniformly characteristic boundary, Comm. Pure Appl. Math. 28 (1975), 607-675.

[10] J. Rauch and F. Massey, Differentiability of solutions to hyperbolic initial-boundary value problems, Trans. Amer. Math. Soc. 189 (1974), 303-318.

[11] S. Schochet, The compressible Euler equations in a bounded domain: Existence of solutions and the incompressible limit, Comm. Math. Phys. 104 (1986), 49-75.

[12] P. Secchi, On nonviscous compressible fluids in a time dependent domain, Ann. Inst. H. Poincaré Anal. Non Linéaire, to appear.

[13] T. Yanagisawa, The initial boundary value problem for the equations of ideal magnetohydrodynamics, Hokkaido Math. J. 16 (1987), 295-314. 